\title{
Author Correction: Lipid nanoparticles for mRNA delivery
}

\section{Xucheng Hou, Tal Zaks, Robert Langer (B) and Yizhou Dong $\mathbb{D}$}

Correction to: Nature Reviews Materials https://doi.org/10.1038/s41578-021-00358-0, published online 10 August 2021.

The definition of DDAB was corrected to dimethyldioctadecylammonium bromide. In addition, the structure of PEG ${ }_{2000}-\mathrm{DMG}$ has been corrected in Figure 2, and its definition in the Figure 2 caption and in the main text has been corrected to 1,2-dimyristoyl-rac-glycero-3-methoxypolyethylene glycol-2000. The definition of $\mathrm{PEG}_{2000}$-DSG has been corrected to 1,2-distearoyl-rac-glycero-3-methoxypolyethylene glycol-2000. These have been corrected both in the PDF and in the HTML versions of the article.

https://doi.org/10.1038/s41578-021-00400-1 I Published online 15 November 2021

(c) Springer Nature Limited 2021 